\title{
Mise en évidence de ruptures de métastabilité par une méthode électrochimique
}

\author{
S. Belcadi $\left({ }^{*}\right)$, J. Bensaid $(* *)$, L. Bougarfa $(* *)$, J. P. Dumas $(* *)$ et A. Jouanneau $\left({ }^{* *}\right)$ \\ (*) Laboratoire de Chimie Physique, Faculté des Sciences, Rabat, Maroc \\ $\left.{ }^{* *}\right)$ Laboratoire de Biophysique, Faculté de Médecine et de Pharmacie, Casablanca, Maroc
}

(Reçu le 28 octobre 1981, révisé le 21 janvier 1982, accepté le 22 février 1982)

\begin{abstract}
Résumé. - On décrit une méthode électrochimique de détection de la cristallisation d'amalgames métastables au cours d'une électrolyse à température fixe. Elle permet de déterminer la fraction molaire de rupture de métastabilité ainsi que la fraction molaire d'équilibre thermodynamique. La méthode a été utilisée dans le cas du binaire mercure-cadmium et contrôlée par analyse Enthalpique Différentielle. A la température ambiante la rupture de métastabilité a lieu à une fraction molaire $12 \%$ supérieure à celle d'équilibre.
\end{abstract}

\begin{abstract}
An electrochemical method of detection of the metastable amalgams crystallization during a monotherm electrolysis is described. It permits to determine the molar fraction of metastability breakdown as well as the molar fraction of thermodynamical equilibrium. The method has been used in the case of mercury-cadmium binary and controlled by Differential Scanning Calorimetry. At ambient temperature, the metastability breakdown takes place at a molar fraction $12 \%$ higher than the equilibrium one.
\end{abstract}

1. Introduction. - Avec le développement des techniques de dosages électrochimiques par redissolution anodique, les chercheurs (par exemple $[2,15,18,19$, 8]) se sont intéressés aux problèmes de la solubilité des métaux dans le mercure. En effet, au cours de la préélectrolyse les concentrations des métaux peuvent devenir importantes et il est possible d'avoir un amalgame saturé à la température de l'expérience. Ceci peut entraîner, lors de la redissolution anodique, une forme des polarogrammes différente de celle obtenue avec des amalgames constitués de solutions vraies. Les caractéristiques (potentiel et intensité maximum, largeur du pic...) ne correspondent pas [19] à celles données par la théorie [17]. Ainsi les valeurs des concentrations de saturation à la température de l'expérience sont-elles utiles à connaître. Toutefois certains auteurs $[8,12]$ signalent l'existence de phénomènes de métastabilité et d'amalgames sursaturés. Mais ces auteurs présentent ces phénomènes comme des "accidents" gênants sans donner de précisions sur leurs résultats.

Or les phénomènes de métastabilité (surfusion, surchauffe...) sont systématiquement rencontrés lorsqu'on étudie des transformations de phases et peuvent être très importants. Par exemple [7], dans certaines conditions, on peut augmenter la concentration d'une solution de $\mathrm{NH}_{4} \mathrm{Cl}$ de $280 \%$ au-delà de la saturation.
Comme il a pu être montré qu'un liquide peut rester surfondu pendant très longtemps $[6,3]$ (plusieurs heures ou plusieurs jours), il est nécessaire de connaitre aussi les concentrations de ruptures de métastabilité. Dans le cas des amalgames rencontrés lors d'expériences utilisant une électrode de travail à base de mercure (électrode à goutte pendante ou à film de mercure) ces valeurs ne sont jamais données. C'est pourquoi nous avons entrepris de mettre au point des méthodes électrochimiques permettant de les déterminer.

Ainsi dans cet article, nous allons décrire les méthodes utilisées pour détecter par voie électrochimique, la cristallisation des amalgames sursaturés mais aussi pour déterminer les valeurs normales des concentrations de saturation. Nous illustrerons ces méthodes par l'étude de la solubilité du cadmium dans le mercure.

2. Phénomènes de rupture de métastabilité. - Généralement les phénomènes de métastabilité et de rupture de métastabilité apparaissent lors de refroidissement : ainsi un liquide, par exemple, peut rester à l'état liquide en-dessous de sa température de fusion $T_{\mathrm{F}}$ (il est surfondu) avant de cristalliser (rupture de métastabilité) à une température inférieure $T$. La rupture de métastabilité est un phénomène aléatoire qui obéit à 
des lois statistiques [20]; ainsi pour différents échantillons identiques d'un même corps, on obtient des températures $T$ différentes, mais on peut définir une température plus probable $T^{*}$ de rupture de métastabilité, les températures $T$ s'écartant peu autour de $T^{*}$.

Les principaux paramètres influant sur $\Delta T=T_{\mathrm{F}}-T^{*}$ le degré de métastabilité, sont : le volume (plus celui-ci est faible, plus $\Delta T$ est important), l'histoire thermique (les résultats sont différents si l'on effectue des cycles refroidissement-réchauffement successifs) ou la vitesse de refroidissement (des phénomènes particuliers apparaissent pour les très grandes vitesses).

Pour illustrer ce qui peut subvenir lors de la rupture de métastabilité dans un amalgame, nous avons schématisé sur la figure 1 une partie d'un diagramme de

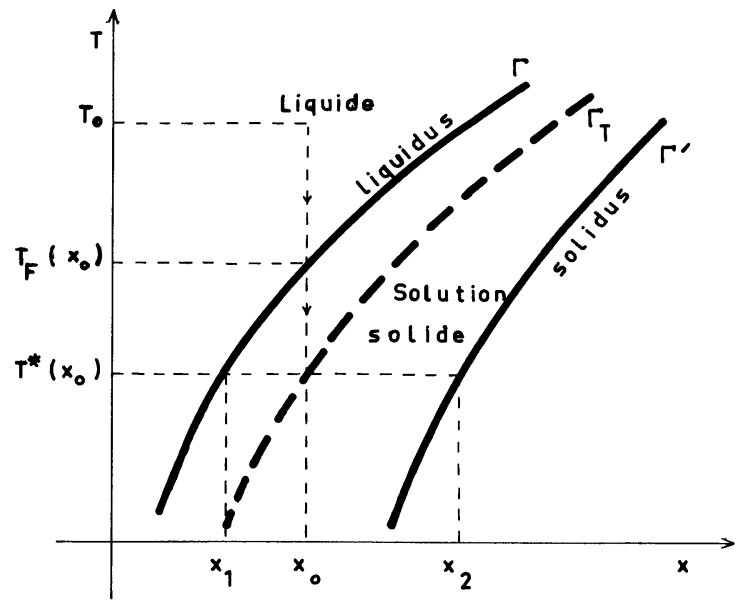

Fig. 1. - Schéma d'une partie du diagramme de phases d'un amalgame (voir texte).

[Scheme of a part of the phase diagram of an amalgam (see text).]

phases dans un domaine de fraction molaire $x$ et de température $T$ où n'existe qu'une solution solide. Sur cette figure 1 la ligne $(\Gamma)$ représente l'équilibre entre la solution liquide et la solution solide (liquidus), la ligne $\left(\Gamma^{\prime}\right)$ représente le solidus.

Si on prend un amalgame de fraction molaire $x_{0}$ à la température $T_{0}$, l'échantillon est entièrement liquide. $\mathrm{Au}$ cours du refroidissement, il devient une solution sursaturée à partir de $T_{\mathrm{F}}\left(x_{0}\right)$. A la cristallisation (rupture de métastabilité), par exemple à $T^{*}\left(x_{0}\right)$, l'échantillon sera constitué d'une partie liquide qui sera une solution de fraction molaire $x_{1}$ et une partie solide qui sera une solution solide de fraction molaire $x_{2}$. Si le dispositif expérimental permet de maintenir $T^{*}\left(x_{0}\right)$ les fractions molaires $x_{1}$ et $x_{2}$ sont imposées par les lignes $(\Gamma)$ et $\left(\Gamma^{\prime}\right)$ (voir Fig. 1). On définit à $x_{0}$ le degré de sursaturation

$$
\Delta T=T_{\mathrm{F}}\left(x_{0}\right)-T^{*}\left(x_{0}\right) .
$$

Si l'on refait l'expérience pour différentes fractions molaires $x_{0}$, on peut définir une ligne appelée courbe d'hypersolubilité $\Gamma_{\mathrm{T}}$ indiquant les températures plus probables de cristallisation en fonction de la fraction molaire.

Supposons maintenant que l'on fasse une électrolyse à température fixe $T_{0}$, d'un amalgame de fraction molaire $x_{0}\left(x_{0}\right.$ pouvant être nulle), la fraction molaire de l'échantillon liquide augmente et la solution devient sursaturée à partir d'une fraction molaire $x_{1}$ telle que $T_{0}=T_{\mathrm{F}}\left(x_{1}\right)$ (voir Fig. 2). A la rupture de métastabilité, par exemple pour une fraction molaire $x^{*}$, il y a apparition d'une phase liquide de fraction molaire $x_{1}$ et d'une phase solide de fraction molaire $x_{2}$. Dans ce type d'expériences monothermes, la rupture de métastabilité est aussi un phénomène aléatoire, les fractions molaires de cristallisation étant différentes d'un échantillon à l'autre mais s'écartant peu d'une fraction molaire plus probable de cristallisation $x^{*}$.

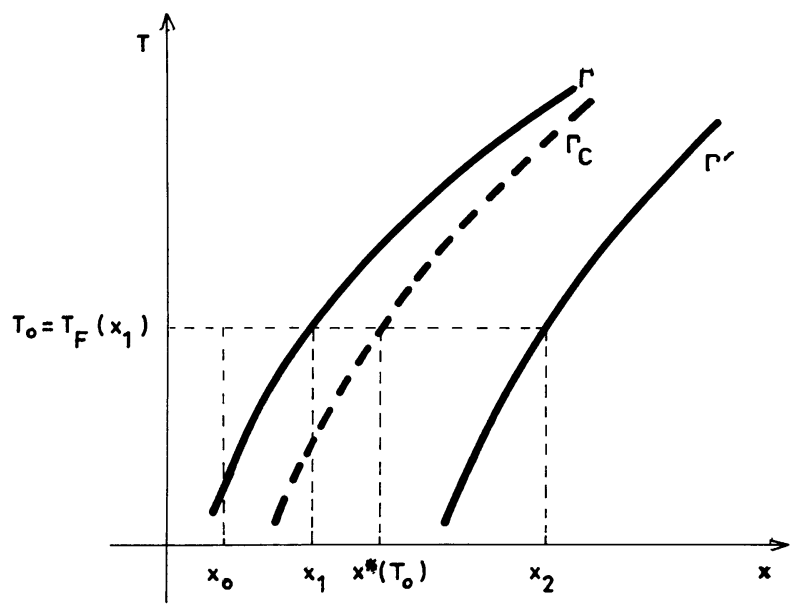

Fig. 2. - Schéma d'une partie du diagramme de phases d'un amalgame (voir texte).

[Scheme of a part of the phase diagram of an amalgam (see text).]

On définit à $T_{0}$ un taux de sursaturation

$$
\Delta x=x^{*}\left(T_{0}\right)-x_{1}\left(T_{0}\right)
$$

et si on fait l'expérience à différentes températures $T_{0}$, on peut définir une courbe d'hypersolubilité monotherme $\Gamma_{\mathrm{C}}$. A priori les courbes $\Gamma_{\mathrm{T}}$ et $\Gamma_{\mathrm{C}}$ sont différentes.

Les techniques expérimentales mises en ouvre pour détecter des ruptures de métastabilité sont essentiellement des techniques visuelles ou calorimétriques. Ce sont ces dernières (par exemple l'Analyse Thermique Différentielle) qui ont été largement utilisées pour les quelques études sur les amalgames [14]. Elles ont l'inconvénient de ne pouvoir être utiles que pour les amalgames où le métal est suffisamment soluble dans le mercure, car, compte tenu des valeurs des enthalpies de dissolution [13], il faut une quantité de phase solide importante pour que la quantité de chaleur apparaissant à la cristallisation soit détectable. D'autre part 
ces techniques ne peuvent être utilisées que lors de refroidissement ce qui n'est pas notre propos.

Ainsi nous allons décrire des méthodes électrochimiques permettant la détection de la cristallisation d'un amalgame et la détermination de $x^{*}$. Toutefois nous donnerons des résultats avec l'amalgame cadmium-mercure où le cadmium est suffisamment soluble dans le mercure pour permettre une étude calorimétrique. Celle-ci servira à tester notre méthode.

3. Détection de la rupture de métastabilité au cours d'une électrolyse. - L'échantillon de mercure ou d'amalgame constitue l'électrode de travail d'un montage potentiostatique classique à trois électrodes. On impose une différence de potentiel égale à $E$ entre l'amalgame et une électrode de référence et on mesure le courant $I$ grâce à la contre-électrode. L'ensemble des trois électrodes plonge dans une solution contenant un sel de métal M. Suivant le sens du courant on aura la réaction

$$
\mathrm{M}^{n+}+\mathrm{Hg}+n \mathrm{e} \rightleftarrows \mathrm{M}(\mathrm{Hg})
$$

dans un sens ou dans l'autre. Afin d'avoir une électrolyse plus efficace nous n'ajoutons pas d'électrolyte support. Nous allons présenter la méthode en supposant que la température est constante.

Imaginons une forme d'échantillon telle que la surface en regard avec la solution soit faible comparée à son volume; dans ce cas la variation de sa concentration est négligeable même s'il y a un courant d'électrolyse. En supposant une vitesse de variation du potentiel faible (pour négliger les effets transitoires dus à la diffusion) on obtient une courbe $I=f(E), E$ variant des potentiels cathodiques vers des potentiels anodiques, de type de celle de la figure 3 .

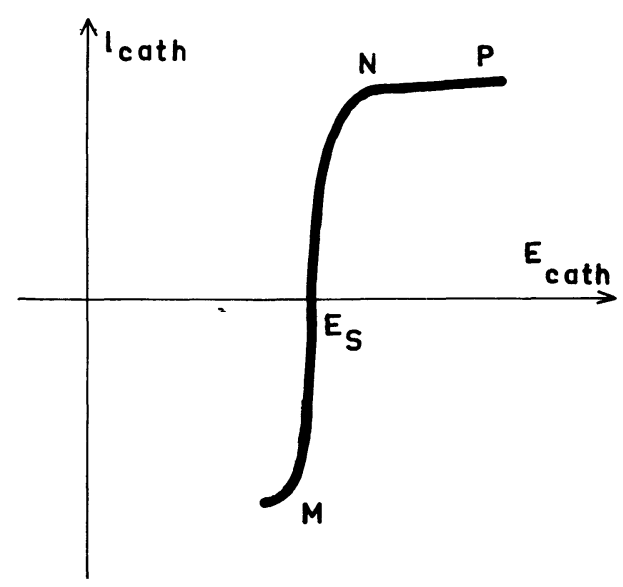

Fig. 3. - Schéma de la courbe « idéale » $I=f(E)$ (voir texte).

[Scheme of the «ideal» curve $I=f(E)$ (see text).]

Sur cette courbe « idéale ", on observe surtout que le courant varie très fortement avec $E$ autour de $E_{\mathrm{S}}$ le potentiel à courant nul. D'autre part, non seulement $E_{\mathrm{S}}$ mais toute la courbe est caractéristique de la fraction molaire de l'amalgame. $E_{\mathrm{S}}$ est donné par une équation de Nernst du type

$$
E_{\mathrm{S}}=E_{0}+\frac{R T}{n F} \log \frac{a^{+}}{a}
$$

$a^{+}$étant l'activité du cation $\mathrm{M}^{n+}$ dans la solution qui ne varie pas sensiblement au cours de l'expérience et $a$ l'activité du métal en solution dans le mercure. Bien que généralement les activités dans le mercure soient différentes des fractions molaires on constate expérimentalement $[8,10]$ que $E_{\mathrm{S}}$ est une fonction affine de $\log x \operatorname{tant}$ que l'échantillon est une phase entièrement liquide. Mais lorsque l'échantillon est cristallisé, la fraction molaire $x$ de la phase liquide est constante et ne dépend que de la température ( $x=x_{1}$ voir Fig. 2). L'activité correspondante $a_{1}$ reste donc constante et le potentiel $E_{\mathrm{S}}$ prend la valeur constante :

$$
E_{\mathrm{SC}}=E_{0}+\frac{R T}{n F} \log \frac{a^{+}}{a_{1}} .
$$

$\mathrm{Si}$ on poursuit l'électrolyse, seule la proportion relative des quantités des phases liquide et solide change.

Sur la figure 4 nous représentons la variation de $E_{\mathrm{S}}$ avec $\log x$ ou plus exactement $\log \alpha$ avec

$$
\alpha=\frac{N_{\mathrm{M}}}{N_{\mathrm{M}}+N_{\mathrm{Hg}}}
$$

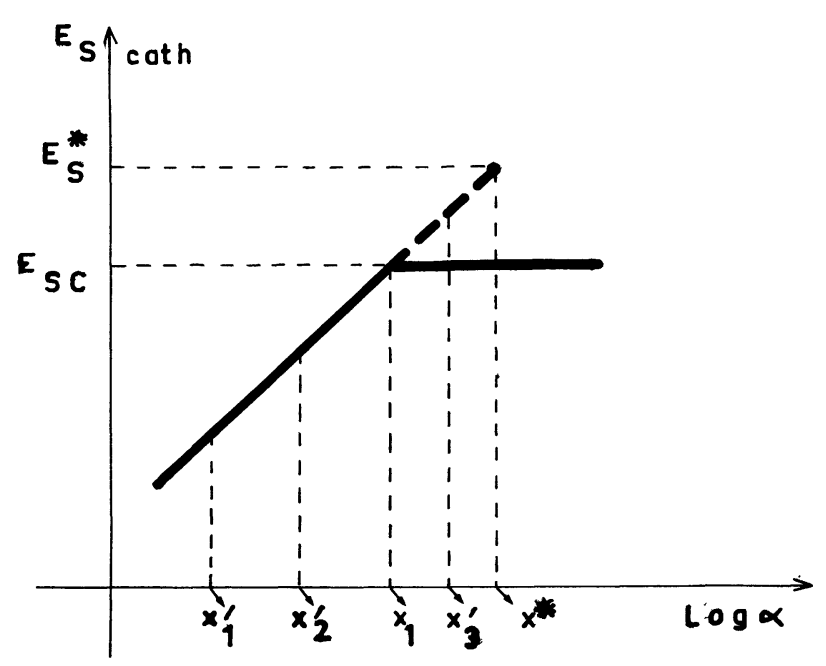

Fig. 4. - Variation de $E_{\mathrm{S}}$ avec $\log \left[N_{\mathrm{M}} /\left(N_{\mathrm{M}}+N_{\mathrm{Hg}}\right)\right]$.

[Variation of $E_{\mathrm{S}}$ versus $\log \left[N_{\mathrm{M}} /\left(N_{\mathrm{M}}+N_{\mathrm{Hg}}\right)\right]$.]

( $N_{\mathrm{M}}$ étant le nombre de moles de métal dans l'amalgame, $N_{\mathrm{Hg}}$ le nombre de moles de mercure) car $\alpha$ n'est égal à $x$ que si tout l'échantillon est liquide.

Le trait plein correspond à des amalgames en équilibre thermodynamique alors que les traits tirés correspondent à des amalgames sursaturés. 
Représentons maintenant sur la figure 5 les courbes "idéales " $I=f(E)$ définies plus haut pour les amalgames de fractions molaires $x_{1}^{\prime}, x_{2}^{\prime}$ (solution liquide stable), $x_{1}, x_{3}^{\prime}$ (solution liquide métastable), $x^{*}$ avant cristallisation, en nous restreignant à la région des courants faibles. De plus $x_{1}^{\prime}<x_{2}^{\prime}<x_{1}<x_{3}^{\prime}<x^{*}$.

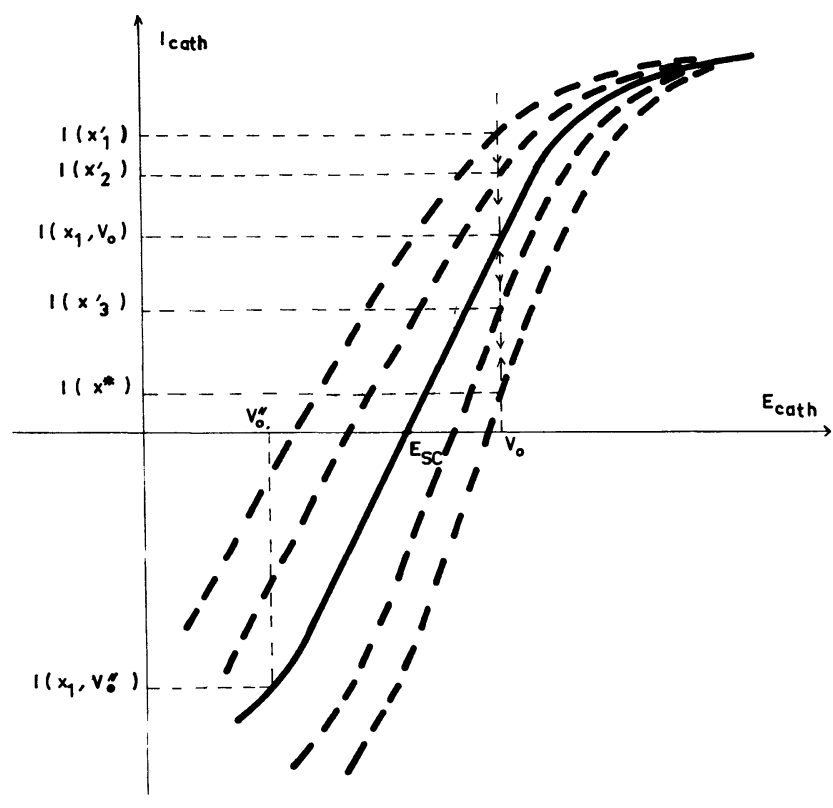

Fig. 5. - Courbes $I=f(E)$ pour différentes valeurs de $x$.

[Curve $I=f(E)$ for different values of $x$.]

Remarquons sur cette figure que lorsque l'échantillon est cristallisé, quelle que soit la quantité de métal dans le mercure, la courbe est celle représentée en trait plein. Par contre, lorsque l'échantillon est liquide, on a une courbe différente pour chaque fraction molaire. Ces courbes se situent au-dessus de la courbe en trait plein pour des solutions stables et en-dessous pour des solutions métastables.

Ainsi si nous prenons un échantillon de dimension finie et que l'on fait une électrolyse à potentiel constant $V_{0}$, la fraction molaire augmentant avec le temps, le courant $I$ diminue jusqu'à $I\left(x^{*}\right)$. Lorsque a lieu la cristallisation, le courant augmente brusquement jusqu'à la valeur $I\left(x_{1}\right)$ où il reste constant. Si on représente sur la figure $6 I$ en fonction de $t$, la durée de l'électrolyse à $V_{0}$, on observe bien un saut de l'intensité.

Ce saut d'intensité est d'autant plus important, relativement, que nous sommes dans la région de variation rapide de $I$ avec $E$ c'est-à-dire proche de $E_{\mathrm{SC}}$ et pour des courants faibles, la sensibilité du polarographe n'étant pas en cause. Par exemple dans le tableau I nous donnons les valeurs de $\Delta I / I$ pour différentes valeurs de $I$ où l'on voit bien que la sensibilité de la méthode est d'autant meilleure que le courant est plus faible

Tableau I

$\begin{array}{llcr}I(\mu \mathrm{A}) & 7,0 & 12,8 & 19,5 \\ \Delta I / I & 0,71 & 0,40 & 0,29\end{array}$

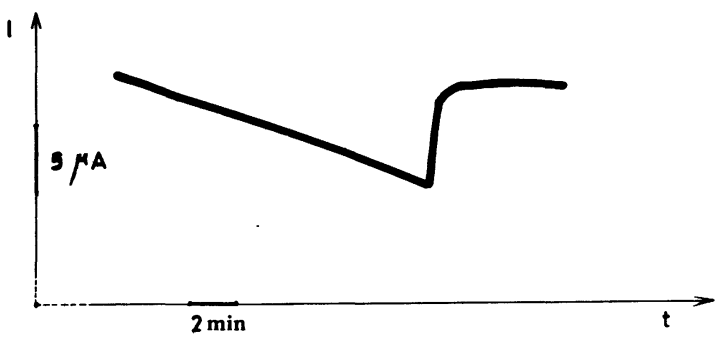

Fig. 6. - Courbe $I=f(t)$ pour $V_{0}<E_{\mathrm{SC}}$ (voir texte). [Curve $I=f(t)$ for $V_{0}<E_{\mathrm{SC}}$ (see text).]

Toutefois, choisir un potentiel $V_{0}$ donnant un courant très faible, augmente la durée de l'expérience, puisque le nombre de réductions pour unité de temps est plus faible et donc $x$ varie lentement.

Ainsi, en pratique, nous effectuons une électrolyse rapide en utilisant un potentiel $V_{0}=V_{0}^{\prime}$ assez cathodique (partie NP de la figure 3 ) de manière à ce que la fraction molaire atteigne rapidement une valeur inférieure mais proche de $x_{1}$ (Fig. 2). Puis nous augmentons $V_{0}$ de manière à se trouver dans la zone $E_{\mathrm{S}}-\mathrm{N}$ (Fig. 3). Nous augmentons la sensibilité car le courant est faible, nous enregistrons $I=f(t)$ jusqu'à ce que l'on détecte la cristallisation et un peu au-delà (voir Fig. 7).

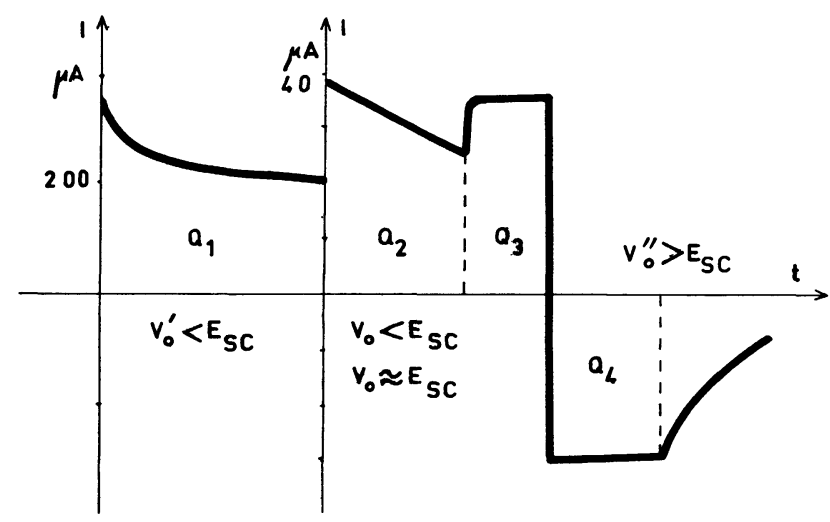

Fig. 7. - Courbe récapitulative d'une expérience (schẻmatique).

[Summarizing curve for an experiment (schematic).]

En intégrant ces courbes $I=f(t)$, on obtient les quantités d'électricité $Q_{1}$ (électrolyse rapide), $Q_{2}$ (jusqu'à la cristallisation), $Q_{3}$ (phase cristallisée).

Le nombre de moles de métal contenues dans le mercure à la rupture de métastabilité est donné alors par :

$$
N_{\mathrm{Mcrist}}=\frac{Q_{1}+Q_{2}}{n F}
$$

$F$ étant le Faraday.

Si on connaît $N_{\mathrm{Hg}}$ (par exemple en déterminant au 0,12 départ les dimensions de l'échantillon de mercure), on 
peut en déduire la valeur de la fraction molaire à la cristallisation $x^{*}$.

Si avec l'échantillon cristallisé, on se place à un potentiel $V_{0}=V_{0}^{\prime \prime}>E_{\mathrm{SC}}$ nous aurons un courant anodique et la quantité de métal dans l'amalgame va diminuer et dans ce cas c'est la quantité de phase solide qui va diminuer. Tant qu'il y aura une parcelle de solide, la fraction molaire de la partie liquide $\left(x_{1}\right)$ restera constante et le courant restera constant (on reste sur la courbe en trait plein de la figure 5). Par contre à partir du moment où il n'y a plus de phase solide, la fraction molaire de la phase liquide diminue (dissolution anodique) et le courant $I$ diminue (en valeur absolue) au fur et à mesure à partir de $I\left(x_{1}, V_{0}^{\prime \prime}\right)$. Ainsi mesurant le courant en fonction du temps, on obtient une courbe du type de celle de la figure 8 .

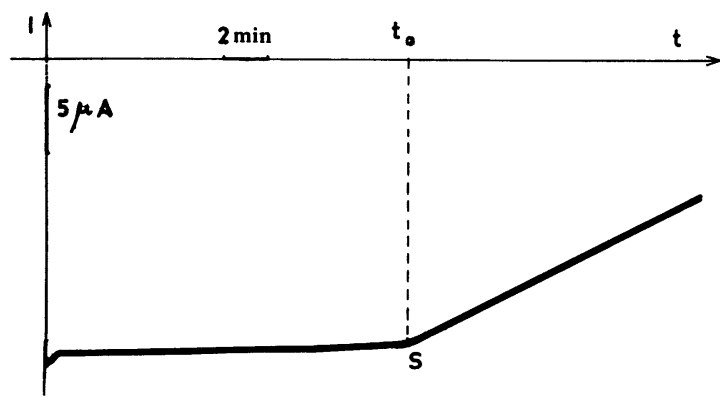

Fig. 8. - Courbe $I=f(t)$ pour $V_{0}^{\prime \prime}>E_{\mathrm{SC}}$ (voir texte).

[Curve $I=f(t)$ for $V_{0}^{\prime \prime}>E_{\mathrm{SC}}$ (see text).]

Sur cette figure le point $\mathrm{S}$ indique l'instant $t_{0}$ à partir duquel il n'y a plus de phase solide, et où la fraction molaire de la solution est égale à $x_{1}$.

Appelant $Q_{4}$ (Fig. 7) la quantité d'électricité mise en jeu au cours de la dissolution anodique jusqu'à dissolution de la dernière parcelle solide, le nombre de moles de métal dans la solution en équilibre à $t_{0}$ est

$$
N_{\mathrm{M}}=\frac{Q_{1} \dot{+} Q_{2}+Q_{3}-Q_{4}}{n F}
$$

et connaissant $N_{\mathrm{Hg}}$ on déduit $x_{1}$.

Travaillant à différentes températures, on peut déterminer la courbe d'équilibre $\Gamma$ dans le diagramme $x, T$ entre la phase liquide et la solution solide (Fig. 1).

4. Techniques expérimentales. - L'échantillon de mercure est constitué d'une goutte accrochée à un fil d'argent. La solubilité du mercure dans l'argent est très grande [16] mais la phase solide (phase $\gamma$ ) ne peut exister que sur une très faible épaisseur car il a été vérifié que la quantité de mercure qui se dissout dans l'argent est négligeable. D'autre part, l'argent est très peu soluble dans le mercure $(0,07$ at. $\%)$ et ne peut avoir d'effet sur les ruptures de métastabilité [5]. La dimension de la goutte est mesurée grâce à un microscope NACHET muni d'un micromètre oculaire.

Le courant est mesuré grâce à un polarographe AMEL 465.
L'électrode de référence est une électrode de calomel saturée et la contre-électrode une électrode de platine. L'oxygène de la solution est enlevé par barbotage d'azote. La température $T_{0}$ est maintenue à $\pm 0,2^{\circ} \mathrm{C}$ par un bain thermostatique JOUAN. La température est mesurée par un thermocouple étalonné. Compte tenu du fait que la cuve d'électrolyse est en pyrex et que la solution utilisée est une solution aqueuse, le domaine de température étudié est limité de 8 à $50^{\circ} \mathrm{C}$ environ. Les quantités d'électricité sont déterminées en mesurant l'aire des courbes $I=f(t)$ grâce à un planimètre.

Le mercure utilisé est un produit Merck de pureté polarographique. La solution aqueuse est une solution de $\mathrm{CdCl}_{2}$ 0,2 M/1 (Merck pour analyses). Celle-ci n'est pas agitée et ne comporte pas d'électrolyte support afin de ne conserver au bout de quelques minutes que le courant de migration qui diminue dans le temps comme il est expliqué dans le chapitre précédent. Cette diminution est surtout sensible lorsque $V_{0}$ est proche de $E_{\mathbf{S}}$ (voir Figs. 7 et 8). La durée de l'expérience est suffisamment importante ( $>30 \mathrm{~min}$.) pour considérer que la concentration du cadmium dans le mercure est homogène.

Afin de confirmer nos résultats, une étude par analyse Enthalpique Différentielle a été entreprise au laboratoire de Thermodynamique de l'Université de Pau (France). L'appareil utilisé est un calorimètre D.S.C. 2 de Perkin Elmer et il a déjà été montré comment cette technique peut s'adapter à l'étude des ruptures de métastabilité $[6,7]$.

5. Résultats. - Comme nous l'avons déjà noté, nous avons choisi pour illustrer la méthode décrite plus haut d'étudier l'amalgame cadmium-mercure car le cadmium est assez soluble $[16,19,4]$ dans le mercure.

Le volume des gouttes de mercure utilisé est de l'ordre de $0,2 \mathrm{~mm}^{3}$. Bien que la réduction des ions $\mathrm{Cd}^{2+}$ puisse se faire avec des réactions intermédiaires [11] nous ne retiendrons que la réaction globale :

$$
\mathrm{Cd}^{2+}+2 \mathrm{e}+\mathrm{Hg} \rightarrow \mathrm{Cd}(\mathrm{Hg}) .
$$

Le diagramme de phases complet [16] indique que dans le domaine de température étudié, nous sommes dans la situation décrite plus haut (Figs. 1 et 2).

Expérimentalement nous obtenons bien des polarogrammes de la forme prévue précédemment (en fait les courbes des figures 6 et 8 sont des courbes expérimentales).

En ce qui concerne la courbe d'équilibre $\Gamma$ entre la solution liquide et la solution solide, nous donnons les résultats sur la figure 9 obtenus par notre méthode électrochimique, par A.E.D. et donnés dans la littérature $[16,19,4]$.

Nous constatons que les résultats sont tout à fait comparables et donc que la méthode que nous avons mise au point permet la détermination de la courbe $\Gamma$ d'équilibre. 


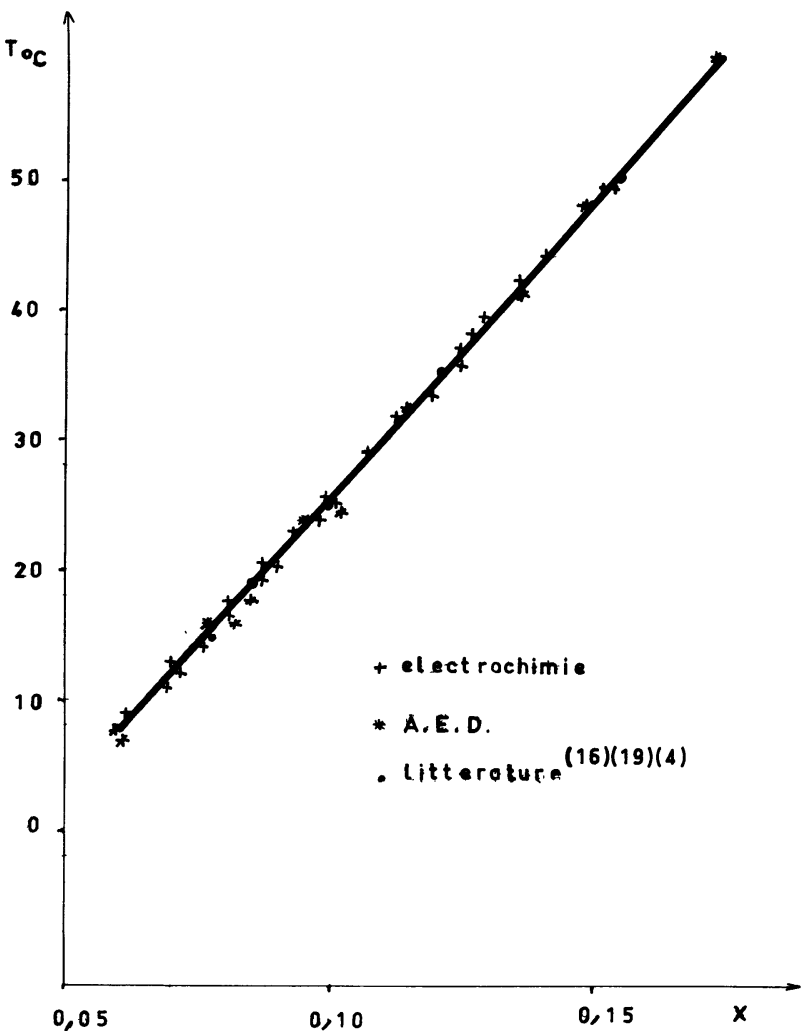

Fig. 9. - Partie du diagramme de phases du binaire $\mathrm{Hg}-\mathrm{Cd}$ déterminé par la méthode électrochimique et par A.E.D.

[Part of the phase diagram of binary $\mathrm{Hg}-\mathrm{Cd}$ determined by the electrochemical method and by D.S.C.]

Sur la figure 10, nous donnons les résultats concernant les ruptures de métastabilité. En réalité ne sont données, en fonction de la température, que les fractions molaires plus probables de cristallisation $x^{*}(T)$. On peut ainsi déterminer la courbe d'hypersolubilité monotherme $\Gamma_{\mathrm{c}}$. On constate que le taux de sursaturation $\Delta x$ diminue légèrement lorsque la température augmente passant de 0,003 à $50^{\circ} \mathrm{C}$ à 0,012 à $10^{\circ} \mathrm{C}$. A $25^{\circ} \mathrm{C}$, température habituelle des expériences électrochimiques, $\Delta x$ pour l'amalgame $\mathrm{Cd}-\mathrm{Hg}$ est de 0,012 soit $\Delta x / x$ de $12 \%$.

$\mathrm{Si}$ nous portons sur cette figure 10 les résultats obtenus par A.E.D. nous constatons que les courbes $\Gamma_{\mathrm{C}}$ et $\Gamma_{\mathrm{T}}$ sont différentes et donc que les retards aux transformations sont plus importants.

Toutefois il faut préciser que, en A.E.D., nous procédons au refroidissement d'une goutte d'amalgame isolée alors que, au cours de nos expériences électrochimiques, la goutte est en contact permanent avec la solution. On peut imaginer que dans ce cas, il y a d'abord formation de particules solides de $\mathrm{Cd}$ à la surface de la goutte de mercure comme le suggèrent des études sur d'autres corps [1, 12, 21]. Ces particules solides favoriseraient la cristallisation (nucléation

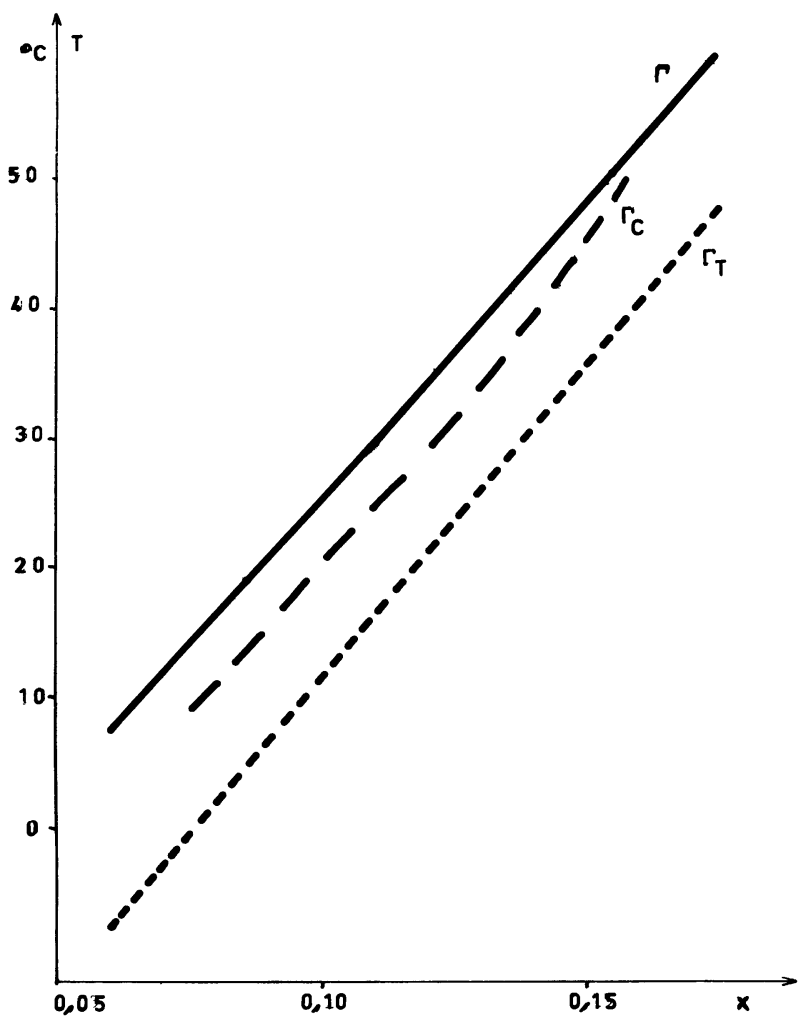

Fig. 10. - Courbes d'hypersolubilité $\Gamma_{\mathrm{C}}$ (méthode électrochimique) et $\Gamma_{\mathrm{T}}$ (A.E.D.) [voir texte] dans le diagramme $(x, T)$.

[Hypersolubility curve $\Gamma_{\mathrm{C}}$ (electrochemical method) and $\Gamma_{\mathrm{T}}$ (D.S.C.) [see text] in the diagram $(x, T)$.]

hétérogène [20]) d'autant plus qu'elles sont constituées du solide qui apparaît à la rupture de métastabilité. Toutefois celles-ci ne sont pas de taille suffisante [20] pour annuler complètement le phénomène de métastabilité.

6. Conclusion. - Dans cet article nous avons décrit des expériences permettant de mettre en évidence des retards aux transformations de phases dans les amalgames. Les méthodes électrochimiques décrites ont permis l'étude des phénomènes de métastabilité dans les conditions habituelles d'utilisation des amalgames comme électrode de travail. D'autre part, il a été possible de déterminer la courbe d'équilibre thermodynamique entre les phases liquide et solide. Les résultats ont été confirmés par Analyse Enthalpique Différentielle. Les expériences sur le binaire mercurecadmium montrent qu'à la température ambiante la fraction molaire de cristallisation est environ $12 \%$ supérieure à la fraction molaire d'équilibre. Les méthodes développées ont une utilisation générale et des travaux sont en cours sur d'autres métaux notamment sur des métaux moins solubles dans le mercure. 


\section{Bibliographie}

[1] Ben Bassat, A. H. I., Azrad, A., Intermetallic compounds formed in mixed (complex) amalgams. I. The systems $\mathrm{CuHg}, \mathrm{ZnHg}$ and $\mathrm{Cu}-\mathrm{Zn}-\mathrm{Hg}$. Electrochimica Acta 23 (1978) 63-69.

[2] Berzins, T., Delahay, P., Theory of electrolysis at constant current in unstrirred solution. II. Consecutive electrochemical reactions. J. Am. Chem. 75 (1953) 4205-13.

[3] Broto, F., Clausse, D., A study of the freezing of super cooled water dispersed within emulsion by differential scanning calorimetry. J. Phys. C. Solid State Phys. 9 (1976) 4251-7.

[4] Claeson, T., Luo, H. L., Anantharaman, T. R., MERriam, M. F., Order-disorder transformations at $2: 1$ composition in the cadmium-mercury system. Acta Metall. 14 (1966) 285-290.

[5] Clausse, D., Contribution à l'étude de la surfusion et de la sursaturation des solutions aqueuses de chlorure d'ammonium. Thèse de Doctorat d'Etat, Pau (1972).

[6] Dumas, J. P., Etude de la rupture de métastabilité et du polymorphisme de corps organiques. Thèse de Doctorat d'Etat, Pau (1976).

[7] Dumas, J. P., Clausse, D., Broto, F., A study of thermograms obtained through differential scanning calorimetry of an emulsion of a supercooled liquid. Thermochimica Acta 13 (1975) 261-75.

[8] Galus, Z., Electrochemical behaviors of metal amalgams, C.R.C. Crit. Rev. Anal. USA 4 (1975) 359422.

[9] Haerdi, W., Buffle, J., Monnier, D., Contribution à l'étude du dosage de submicrotraces de fer par polarographie inverse sur goutte de mercure pendante I. J. Electroanal. Chem. 23 (1969) 81-88.

[10] JANGG, G., KIRChMAYR, H., Das elektrochemish Potential der amalgame. Z. Chem. 2 (1963) 47-56.
[11] Jovanovic, D. R., RaKic, T., On the kinetics and mechanism of the $\mathrm{Cd}(\mathrm{II}) / \mathrm{Cd}(\mathrm{Hg})$ system in acid media. Electrochimica Acta 22 (1977) 233-236.

[12] Kemula, W., Galus, Z., Bull. Acad. Pol. Sci. Ser. Sci. Chem. 7 (1959) 729.

[13] Marco, F., Navarro, J., Torra, V., Application of flow calorimetry to the study of alloy formation. I. Enthalpy of solution In, $\mathrm{Cd}, \mathrm{Zn}, \mathrm{Pb}, \mathrm{Ca}, \mathrm{Sn}$ and $\mathrm{Bi}$ in $\mathrm{Hg}$ at 293.15K. J. Chem. Thermodynamics 7 (1975) 1059-1066.

[14] Monge, P., Contribution à l'étude de la surfusion du mercure. Thèse de doctorat d'Etat, Bordeaux (1968).

[15] Murray, R. W., Gross, D. J., Chronopotentiometric and chronocoulometric measurements of absorption of lead and mercury (II) at mercury electrodes. Analytical Chemistry 38, 3 (1966) 392-404.

[16] Pascal, P., Nouveau traité de Chimie minérale, Tome XX, Alliages métalliques (Masson Ed.) 1963.

[17] Roe, D. K., Toni, J. E. A., An equation for anodic stripping curves of thin mercury-film electrodes. Analytical Chemistry 37, 12 (1965) 1503-6.

[18] Shain, I., Lewinson, J., Stripping analysis with spherical mercury electrodes. Analytical Chemistry 33, 2 (1961) 187

[19] StojeK, Z., StePnik, B., Kublik, Z., Cyclic and stripping voltammetry with graphite based thin mercury film electrode prepared "in situ ». J. Electroanal. Chem. 74 (1976) 277-295.

[20] Turnbull, D., Phase changes. Solid State physics. (Acad. Press. Inc. Publishers, New York) 1956, 2 , 226-306.

[21] Verplaetse, H., Donche, H., Temmerman, E., VerBEEK, F., Study of the solubility of antimony in mercury by cyclic and stripping voltammetry. J. Electroanal. Chem. 93 (1978) 213-219. 